\title{
De novo individualized disease modules reveal the synthetic penetrance of genes and inform personalized treatment regimens
}

\author{
Taylor M. Weiskittel, Choong Y. Ung, Cristina Correia, Cheng Zhang, and Hu Li \\ Center for Individualized Medicine, Department of Molecular Pharmacology and Experimental Therapeutics, Mayo Clinic \\ College of Medicine, Rochester, Minnesota 55905, USA
}

\begin{abstract}
Current understandings of individual disease etiology and therapeutics are limited despite great need. To fill the gap, we propose a novel computational pipeline that collects potent disease gene cooperative pathways to envision individualized disease etiology and therapies. Our algorithm constructs individualized disease modules de novo, which enables us to elucidate the importance of mutated genes in specific patients and to understand the synthetic penetrance of these genes across patients. We reveal that importance of the notorious cancer drivers TP53 and PIK3CA fluctuate widely across breast cancers and peak in tumors with distinct numbers of mutations and that rarely mutated genes such as XPOI and PLEKHAl have high disease module importance in specific individuals. Furthermore, individualized module disruption enables us to devise customized singular and combinatorial target therapies that were highly varied across patients, showing the need for precision therapeutics pipelines. As the first analysis of de novo individualized disease modules, we illustrate the power of individualized disease modules for precision medicine by providing deep novel insights on the activity of diseased genes in individuals.
\end{abstract}

\section{[Supplemental material is available for this article.]}

Pooled -omic data from patient samples have enabled construction of cellular interaction modules that provides a system-level understanding of disease etiology. This new conceptualization of disease has led to discoveries of previously unknown mechanisms and has significantly expanded opportunities for therapeutic targeting (Iborra-Egea et al. 2017; Sharma et al. 2018). Specifically, system and network science has pinpointed novel pharmacological targets and opportunities for drug repurposing or drug-drug synergies (Zhao and Iyengar 2012). Advancements owing to system biology have been even more pronounced in oncology. The reconstruction of complex cancer disease modules describes tumor biology at the system level, which is particularly important for such a polygenic and dynamic disease (Zielinski et al. 2017; Lin et al. 2019).

Despite these recent advancements, a truly individualized system approach has yet to be applied to individualized medicine. Oncology patients in particular experience highly variable disease phenotypes and drug responses. The need for precision approaches in oncology has therefore been well established, with numerous scientists and clinicians calling for innovation (Aronson and Rehm 2015; Relling and Evans 2015; Carrasco-Ramiro et al. 2017; Werner et al. 2017). Patient-derived xenograft (PDX) models and clinical studies have highlighted the heterogeneity of tumor mechanistic properties and therapeutic responses (Chiron et al. 2014; Dagogo-Jack and Shaw 2018; Xu et al. 2019). Some of this variability can be captured with patient stratification through disease subtype classification or biomarker testing, but the majority of inter-patient variability remains unexplained (Dagogo-Jack and Shaw 2018). The lack of broadly applicable biomarkers indicates

\section{Corresponding author: li.hu@mayo.edu}

Article published online before print. Article, supplemental material, and publication date are at https://www.genome.org/cgi/doi/10.1101/gr.275889.121. Freely available online through the Genome Research Open Access option. that unique system-level interactions are at play within single patients. System and network biology is poised to capture these phenomena well, but new theoretical frameworks and computational approaches must be implemented to make such precision network biology a reality.

Existing methodologies extract disease modules (or "disease networks"), which are highly perturbed subnetworks of the larger cellular interactome where disease gene interactions occur (Menche et al. 2015). Previous approaches have attempted to detect and prioritize individualized cancer drivers, but these algorithms infer their individualized analyses from cohort-level disease modules (Bashashati et al. 2012; Cho et al. 2016; Reyna et al. 2018). For example, the LIONESS algorithm uses aggregate disease modules generated by existing approaches to linearly interpolate individual sample modules (Kuijjer et al. 2019). We hypothesize that although some individual patient disease activity is recapitulated in the cohort disease modules, there are additional unexplored interactions detectable only at the individual patient level, which dictate patient-specific mechanisms, phenotypes, and therapeutic responses. We additionally suspect that at the gene level, there are patient-specific variations in pathogenicity. This is because patients possess highly varied basal cellular environments and mutations (The Cancer Genome Atlas Network 2012; Dagogo-Jack and Shaw 2018). Given that current approaches rely heavily or exclusively on cohort-inferred disease modules, we suspect that inter-patient variability and precision have been underrepresented.

Although practical, using features inferred across the cohort fail to capture patient individualized features by disregarding rare unique factors within a patient. A new approach is needed to truly

(C) 2022 Weiskittel et al. This article, published in Genome Research, is available under a Creative Commons License (Attribution-NonCommercial 4.0 International), as described at http://creativecommons.org/licenses/by-nc/4.0/. 
infer individualized disease modules that accurately recapitulate individualized disease. In this study, we examined on the collective actions of mutated genes to try to understand individualized disease at a deeper level. We hypothesized that cohort disease modules are poorly representative of individualized disease and that new insights in precision medicine would reveal themselves once we zoomed in on individual patients. Thus, we set out, first, to create a robust pipeline for individualized disease module construction and, second, to use these disease modules to characterize individualize disease pathobiology and therapeutics.

\section{Results}

Shortest-path analysis of individual patient mutated genes encapsulates disease activity into individualized disease modules

Rather than occupying an average disease module, we predicted that individual disease modules likely occupy their own distinct foci within the larger proteinprotein interactome (Fig. 1A; Menche et al. 2015). We thus set out to develop a pipeline for the construction of single-patient disease modules. Disease modules have historically been inferred at the cohort level using correlative, random walk, or shortest-path approaches (Codling et al. 2008; Managbanag et al. 2008; Cerami et al. 2010; Chen and Zhang 2013; Cahan et al. 2014; Jia and Zhao 2014; Da Rocha et al. 2016). Because disease modules are generally dense and scale-free collections of interacting disease genes, (Wuchty 2001) shortest-path analysis has proven to be a robust tool for network analysis in biological settings (Yu et al. 2007; Managbanag et al. 2008; Chen et al. 2016). Shortest-path analysis also offers technical advantages over the other approaches. Correlative approaches require numerous samples for each patient, which is very rare, particularly in the multiomic setting. On the other hand, random walk analysis is extremely computationally intensive, requires several iterations to reach a consensus, and is only a locally optimized search strategy (Xia et al. 2020). Furthermore, shortest paths have been found to be the strongest and most likely interaction pathways in other applications of network topology (Katzav et al. 2015). We therefore began to build individual disease modules with a shortest-path approach on a protein-protein interaction (PPI) network (Fig. 1B). For our individualized analysis, a shortest-path approach allowed us to connect sparse unique sets of diseased genes. We used the well-documented PPI iRefIndex containing numerous categories of wellvalidated protein-protein interactions to find these pathways
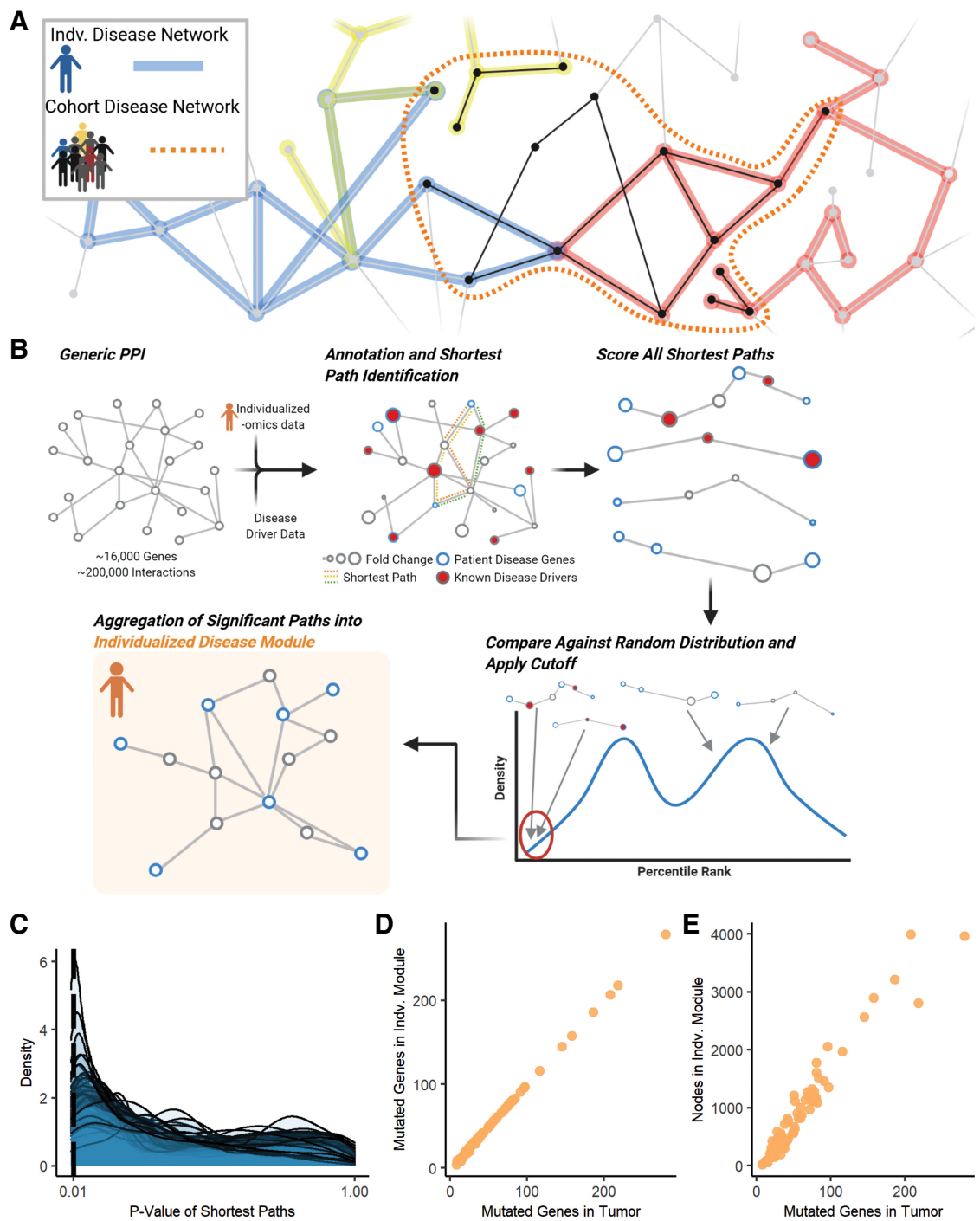

Figure 1. Individualized disease module concept and construction pipeline. (A) Schematic illustrating how individualized disease modules are related to cohort-inferred disease modules. $(B)$ Our construction pipeline begins with annotation of a generic protein-protein interaction (PPI) network with disease-context and individualized omics data. Following annotation, all shortest paths between diseased genes are and evaluated for disease activity. These paths are compared with randomly generated path ways via empirical $P$-value. Pathways that are less than empirical $P$-values of 0.01 are added to the individualized disease modules. (C) Density plots displaying the distribution of $P$-values for each patient's rea paths. $(D, E)$ Scatter plot of the number of mutated genes $(D)$ and nodes $(E)$ in the individualized disease modules versus the number of mutated genes in the tumor.

(Razick et al. 2008). For this reason, we favored iRefIndex over experimental databases that typically only capture one mode of interaction (Luck et al. 2020) and are a fraction of the size. The other database we considered was the STRING database, which is larger than iRefIndex but contains numerous interactions that are not limited to physical interactions and is less stringently validated.

We began with 90 breast cancer (BC) patients from the TCGA BRCA Project as a proof-of-concept cohort for our precision system biology pipeline (Supplemental Table S1; The Cancer Genome Atlas Network 2012). These patients were of diverse mutational burden and PAM50 subtype, thus capturing the biological heterogeneity of the BC (Supplemental Fig. S1A). We designated mutated genes identified by TCGA mutation annotations as "diseased genes." These included missense, nonsense, insertion, and 
deletion mutations, which were detected using Sniper, MUTECT, VarScan, and Muse (Koboldt et al. 2012; Larson et al. 2012; Cibulskis et al. 2013; Fan et al. 2016). We then found all shortest paths between all pairs of diseased genes for each individual patient (Fig. 1B; Supplemental Fig. S1B). We then enriched for paths likely to contain disease activity with three parameters. First, we measured gene expression fold change of each gene within a path because proteins with pathogenic mutations are known to frequently cause transcriptomic changes in interacting partners (Zhong et al. 2009). Second, in order to capture multigene interactions, the shortest paths that crossed a third diseased gene were prioritized. Finally, paths that contained established cancer drivers were also prioritized because minor or rarer disease actors have been found to encourage pathogenic activity through known cancer drivers (Castro-Giner et al. 2015; Sondka et al. 2018).

The probability density function of patient paths compared with 1000 random paths of equivalent length indicated a highly significant subset of paths as measured by empirical $P$-value (see Methods). These significant paths were aggregated together to form disease modules for each patient (Fig. 1B; Supplemental Fig. S1B). To understand how differing $P$-value cutoffs affect the pipeline, we selected five patients of varying mutational burdens and recorded network parameters as we varied the $P$-value cutoff. As expected, the number of significant paths and network size increased with increasing $P$-values (Supplemental Fig. S2A-C). The increases seemed to be exponential, indicating that stringent $P$-values are needed to keep modules small and enriched in the most important diseased genes. Given these results, we moved forward with a $P$-value cutoff of 0.01 , which provided a good balance of network size and stringency. In our entire cohort, we saw a subset of highly enriched paths for each patient out of the numerous paths that were detected (Fig. 1C; Supplemental Fig. S2D-F). Approximately $5 \%-15 \%$ of detected paths were ruled significant using the cutoff of 0.01 (Supplemental Fig. S2F). Using this process, we were able to generate individualized disease modules for each of the 90 patients without cohort-inferred features.

The properties of patient disease modules (Supplemental Table S2) were analyzed to understand the implications of the number of disease genes (mutational burden) on the resultant network. Almost all mutations were carried forward to the individualized disease modules (Fig. 1D; Supplemental Table S3). This indicates that even mutations that would normally be considered "passengers" can have impactful effects when cooperating with co-occurring "drivers." This result is explained by the "mini-driver model," which postulates that, in cancer, disease genes lie on a spectrum from driver to passenger (Castro-Giner et al. 2015). Under this model, individualized patient context (mutated genes, underlying genetics, transcriptome) dictates where on this spectrum a disease gene lies. Our work supports this model because we observe that minor mutations can be implicated in disease module activity through their interactions with more substantial cancer drivers. Patients with an increased mutational burden had larger individualized disease modules as measured by edge and node number (Fig. 1E; Supplemental Fig. S3A; Supplemental Table S3). With the addition of each mutation, more nondiseased genes are brought into the disease module.

We next compared individualized disease modules to a cohort-based module to confirm our hypothesis that the cohort module poorly recapitulates individualized disease (Fig. 1A). A cohort disease module was generated using the GRNBoost algorithm and all patient transcriptomic profiles (Moerman et al. 2019). We found that for most patients only half of their individualized mod- ule nodes were represented in the cohort module (Supplemental Fig. S3B). Furthermore, we saw that only $60 \%-20 \%$ of individual patient mutations were included the cohort module and that there were thousands of extra nodes in the cohort module (Supplemental Fig. S3C,D). To understand if there was a bias of cohort modules toward frequently mutated genes, we inspected the percentage of rare versus frequent mutations carried over to the cohort module in each patient (Supplemental Fig. S3E). A discernable bias was not detected when looking at the percentage of excluded rare and frequent mutations, but numerically many more rare mutations were left out of the cohort disease module per patient, which is not unexpected because rare mutations are more frequent (Supplemental Fig. S3F). Cumulatively, these results confirm that cohort disease modules are not representative of many features of individualized disease.

\section{In silico disruption of individualized disease module identifies personalized mutated driver genes}

With individualized disease modules established, we sought to understand the varying importance of disease genes for each patient. To quantify disease gene importance, we measured the changes in the network parameters hubs, bottlenecks, network components, and edges when a disease gene and its cooperative paths are removed from the individual disease module (Fig. 2A; Supplemental Fig. S4A-E). Here we hypothesize that changes in network parameters upon gene removal can give insights into how crucial a given gene is to the overall disease module and ultimately the overall disease phenotype. Thus, the network components were aggregated into a quantitative score of individualized disease module disruption that we termed individualized disease gene importance (IDGI) (Fig. 2A).

Because this score was generated to assess disease genes in individual patients, we first validated IDGI by comparing real and randomized individualized patient disease modules. Randomizing trials were completed 10 times in 10 patients for a total of 100 separate trials. High-scoring disease genes in real disease modules scored significantly above shuffled disease modules, thus confirming the IDGI methodology (Supplemental Fig. S5A-J). Furthermore, IDGI was able to recover genes listed by the Cancer Gene Census (CGC) as implicated in cancer pathobiology with an AUC of 0.821 and 0.879 for all cancers and breast cancer, respectively (Supplemental Fig. S6A; Sondka et al. 2018). Using the same five test patients and CGC recovery from Supplemental Figure $\mathrm{S} 2$, A through $\mathrm{C}$, we completed an ablation experiment to understand each network components' effect on the IDGI score. Edge number was the most important component as shown by the strongest decrease in AUC (Supplemental Fig. S6B-D). The other IDGI components did not markedly decrease AUC when removed, but together they were able to maintain a reasonable AUC when edges were removed, indicating that these collectively contributed to an AUC increase (Supplemental Fig. S6B-D). With the IDGI score well validated, we returned to the path selection scheme to understand how each component contributes to individualized disease module construction. Again, using the five test patients and the CGC recovery validation scheme, we found that disease information contributed the most to AUC followed by patient mutations (Supplemental Fig. S6E,F). This is not unexpected because this validation scheme only tests the pipeline's ability to recover known disease drivers, and thus, it is an imperfect test of our pipeline because we seek to identify rare and frequent drivers in an individualized manner. We retained the individualized omic

\section{Genome Research}

www.genome.org 


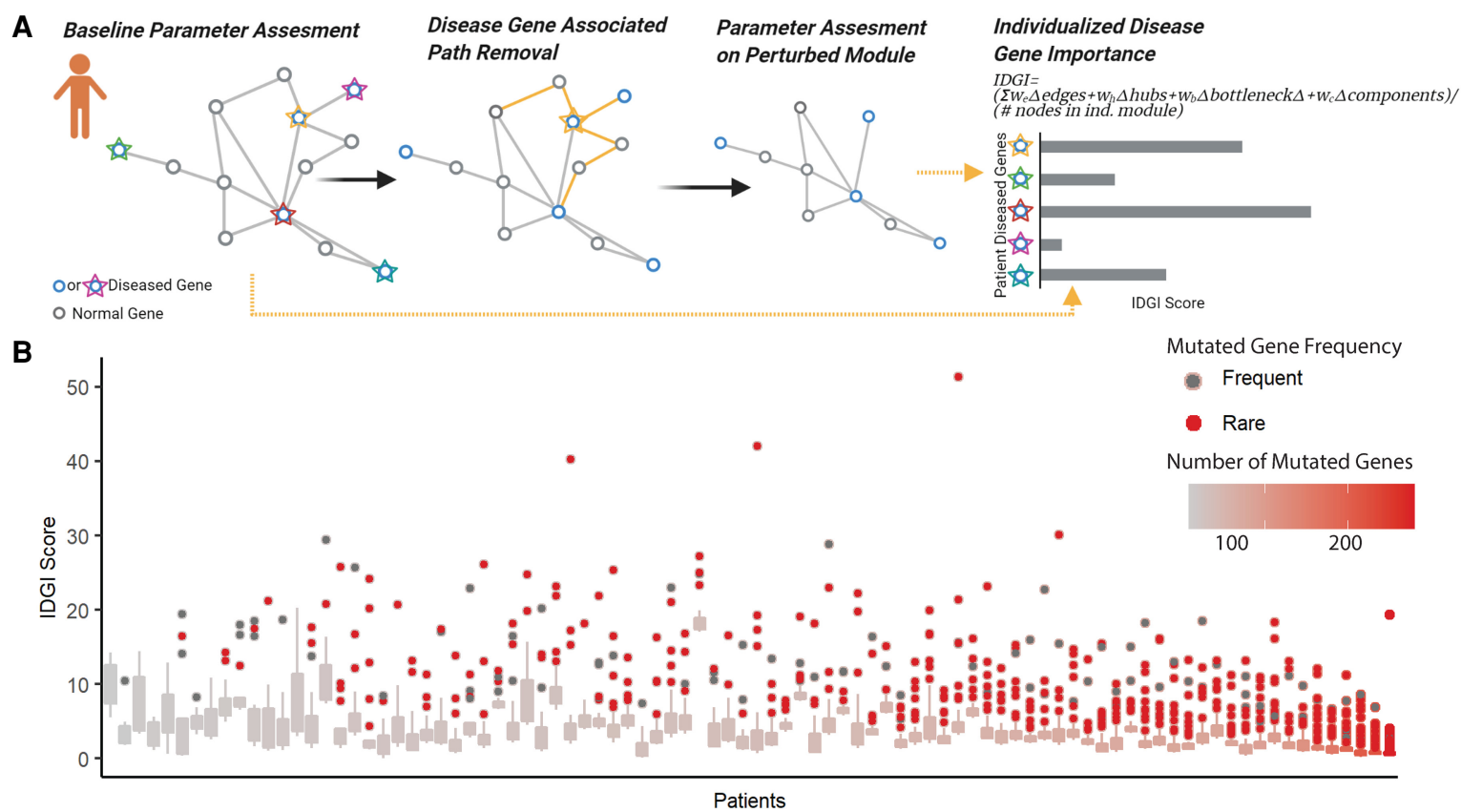

Figure 2. Disease gene importance scoring process and scoring results across patients. ( $A$ ) The individualized disease gene importance (IDGI) scoring pipeline begins with the accessing the baseline parameters of the individualized disease modules. Next the disease gene is scored, and the shortest paths associated with the disease genes are removed from the individualized disease module. The change in parameters is then quantified into the IDGI score. (B) Boxplots showing the distribution of IDGI scores for each patient. High IDGI scoring genes (outliers) are colored to show rare $(<5 \%$ mutated in TCGA BC) and frequent mutations ( $>5 \%$ mutated in TCGA BC).

features of fold change and mutation status because of this in our final pipeline. Nevertheless, these validation in silico experiments together confirmed that our method provided individualized and disease relevant quantitation.

Looking at the IDGI scoring for each patient, several highscoring outlier disease genes were noted in most patients. Each patient's outliers were a mix of rarely $(<5 \%$ in the TCGA BRCA cohort) and commonly ( $>5 \%$ in the TCGA BRCA cohort) mutated genes (Fig. 2B; The Cancer Genome Atlas Network 2012). This finding affirms the need for an individualized approach as many of the rarely mutated genes would likely not be detected in statistical or cohort-based analyses. We next integrated gene scores with patient disease modules to visualize disease gene importance. In some patients, well-known drivers such as PIK3CA carried high importance (Fig. 3A; Supplemental Fig. S7A,B), and other patient disease modules were instead driven by rare mutations. In one example, PIK3CA and TP53 play a secondary role to the rarely mutated genes XPO1 and PLEKHA1 (Fig. 3B; Supplemental Fig. S8A,B). $X P O 1$ was of particular interest because an XPO1 inhibitor induced complete remission in a limited number of patients during solid tumor clinical trials (Azizian and Li 2020). Furthermore, PLEKHA1, a largely uncharacterized membrane signaling protein, has not been described in BC thus far (Dowler et al. 2000). Both genes are mutated in $<1 \%$ of BC cases (The Cancer Genome Atlas Network 2012). To display all 90 patients' data, we created an interactive data portal (Supplemental Table S4; https://syspharm
A

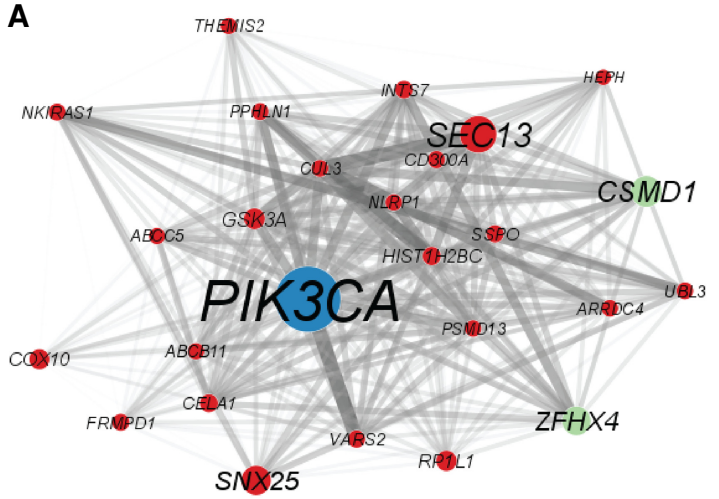

B

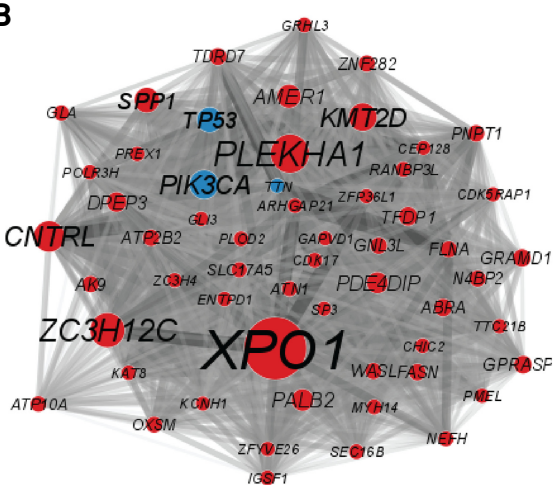

Mutation Frequency

$>15 \%$

5-15\%

$<5 \%$

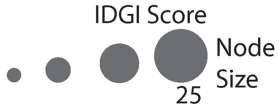

Figure 3. Individualized disease modules displaying varied gene importance in two representative patients. $(A, B)$ Individualized disease modules for two representative patients. Edge color intensity is determined by the distance between the mutated genes. Node size corresponds to IDGI score, and node color reflects mutation frequency within the entire TCGA BRCA cohort. 
.shinyapps.io/PERMUTOR/). Each of these patients exemplify the unique potential of precision system analysis that allows for the highlighting of rare important mutations that we predict drive patient-specific disease.

Synthetic penetrance describes the varying importance of disease genes across patients

We further appreciated the varying influence of known disease genes when we examined them across our entire cohort. Here we found that, irrespective of cancer subtype, scores varied drastically across patients. TP53, PIK3CA, CDH1, GATA3, and MAPK subunit genes scored the highest on average; TP53 and PIK3CA showed a broad range of importance across the cohort (Fig. 4A,B). TTN mutations, which are common but largely inconsequential, were the third most frequent in our cohort (Oh et al. 2020). IDGI scores for TTN revealed low scoring across all patients, which indicates that our IDGI scoring scheme can detect "passenger" genes (Fig. 4B).

We next examined the scores of PIK3CA and TP53 to characterize how IDGI varied with tumor properties. Seven patients had mutations in both of these genes, and in five of the seven, both genes had a low IDGI, indicating a diffusion of disease activity to lesser-known mutated genes. PIK3CA, the second most frequently mutated gene in breast cancer, had the highest level of importance in patients with a lower mutational burden (fewer than 30 mutated genes) (Supplemental Fig. S9A). Conversely, the most commonly mutated gene, TP53, was more important in patients with a higher mutation burden (30 to 100 mutated genes) (Supplemental Fig. S9B). TP53 IDGP showed more variable activity in missense mutations compared with splice site mutations (Supplemental Fig. S9C). Classifying missense mutations as in or outside of known mutational hotspots reveals that mutations within hot- spots contribute more to the disease module on average (Supplemental Fig. S9D; Baugh et al. 2018).

The presence of unique cancer drivers is often hypothesized, and our gene importance scoring reveals an additional aspect of this postulate. As we have shown, commonly mutated genes vary in importance in different patients. We term this variable impact of disease genes across patients "synthetic penetrance" (Fig. 5). Synthetic lethality was conceptualized following the observation that, in some model organisms and cancer cell lines, specific combinations of genetic perturbation result in cell death $\left(\mathrm{O}^{\prime} \mathrm{Neil}\right.$ et al. 2017). As in synthetic lethality, here "synthetic" describes the requirement of specific settings and partners to enable disease gene activity, and "penetrance" encapsulates the variability in gene importance among individuals. Our examination of commonly mutated genes within BC illustrates that synthetic penetrance is influenced by known biological contexts and is an important factor in precision medicine.

\section{Simulation and prioritization of personalized therapeutic targets via disease module disruption}

We next used disease module disruption to prioritize therapeutic targets. Target therapies are an emerging class of pharmaceuticals that are aimed at specific genes that cancer cells use for invasion or survival. We examined the disease module importance of FDA-approved target-therapy genes (Supplemental Table S5) in individual patients using an adapted gene importance score termed individualized target gene importance (ITGI). BC target-therapy genes showed significantly elevated importance over those used in other cancers (Fig. 6A; Supplemental Table S6). When examining each gene specifically, we found that BC target-therapy genes ranged in disease module disruption efficacy among individuals (Fig. 6B). Surprisingly, some target-therapy genes from other cancers had high disruptive potential for certain patients (Fig. 6C; Supplemental Fig. S10). For example, BTK (zanubrutinib approved for mantle cell lymphoma) and MAPK1 (trametinib approved for melanoma) were low scoring on average but scored with high disease module importance in one patient each despite not being clinically approved BC targets (Fig. 6C; Wishart et al. 2008). Neither gene was mutated in these patients, which confirms the need for an individualized system approach because such targets could not be identified with genomics alone.

As with IDGI scores (Fig. 2B), each patient had high-scoring ITGI outliers, but the identity of top target-therapy genes was highly variable from patient to patient (Supplemental Fig. S11A-D). $\mathrm{BC}$ target genes can be further classified by FDA approval for specific BC subtypes. When the patient cohort was divided by BC subtype, the target-therapy genes scored higher on average for their approved subset of patients (Supplemental Fig. S11E-I). We added the target analysis to the data portal for further exploration of individual patients (Supplemental 


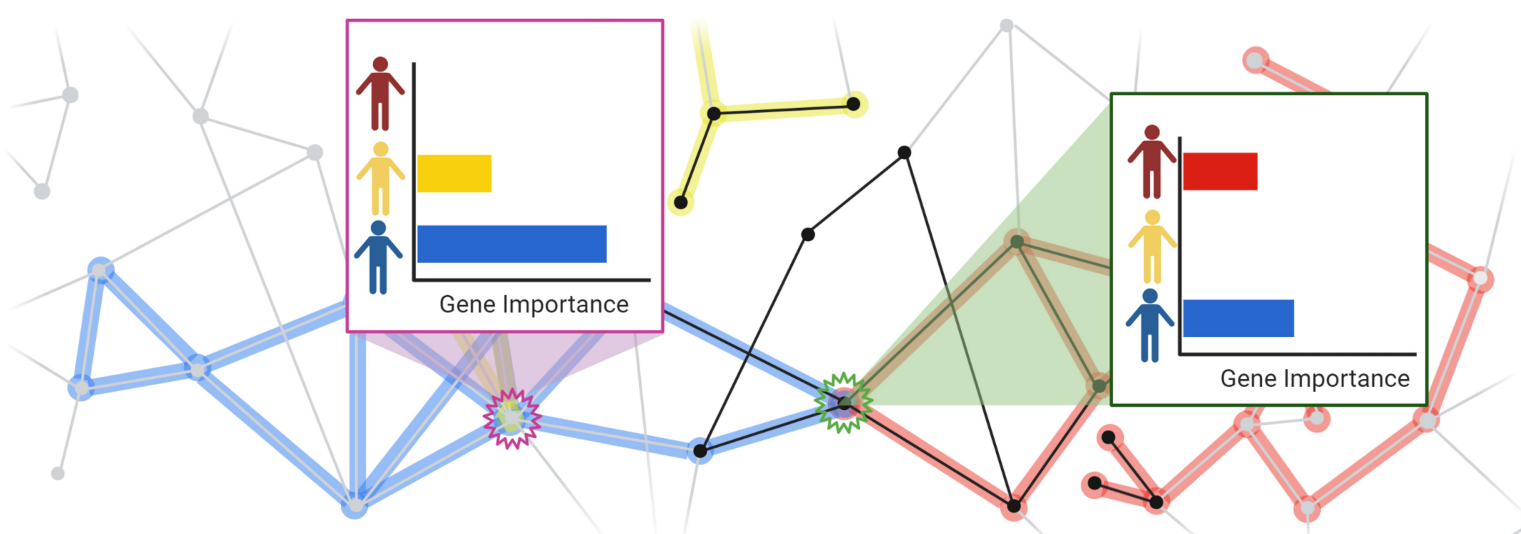

Figure 5. Synthetic penetrance of disease genes within individualized disease modules. Schematic showing the concept of synthetic penetrance in three individualized disease modules (red, blue, and yellow). Synthetic penetrance is shown for two representative genes (pink and green) by showing the varied importance of disease genes in different patients.

Table S6; https://syspharm.shinyapps.io/PERMUTOR/). Across these analyses, group behavior fell in line with conventional drug approvals, but individualized examination of ITGI scores revealed that there are important patient-specific variations that should be accounted for in precision medicine approaches.

Oncology drugs are often used in combination during cancer patient treatment (Zagidullin et al. 2019). Predicting efficacious combinations of approved drugs is a persistent clinical need, and devising patient-specific combinatorial regimens is an additional challenge that must be addressed for next generation therapeutics (DiNardo and Perl 2019). We reason that ITGI scoring could be applied to reveal potential combinatorial treatment regimens for single patients. All but one patient showed an increase in maximum ITGI score with an optimal target pair compared with the highestscoring single target (Fig. 6D; Supplemental Table S7). Combinatorial gene scores fell into three categories: less than additive (overlapping) single-target scoring, equal to additive scoring, and higher than additive scoring (synergistic). We identified improved synergistic pairs for patients who had low single-target scores (Fig. 6E,F; Supplemental Fig. S12A,B). In other cases, target-therapy combinations showed far less than additive disease module disruption, indicating redundancy in targeting (Supplemental Fig. S12C-F). Such redundancy in therapeutic coverage may be favorable depending on biological context. Our results show the success and feasibility of using disease module disruption for individualized therapeutic regimens and drug repurposing.

\section{Discussion}

Disease modules have become a vital tool for disease-understanding cohort studies. Here we show that disease modules reveal even deeper meaning when applied at single-patient resolution. Numerous calls for new approaches in precision medicine have been put forward by the oncology community, but marginal progress has been made (Relling and Evans 2015; Werner et al. 2017; Zhu et al. 2019; Yadav et al. 2020). This work adds to the toolset of clinicians and scientists in precision medicine by enabling the construction and functionalization of patient-specific disease modules. We anticipate that this approach will be generalizable to other cancers and even to other categories of polygenic disease such as metabolic and neurodegenerative disorders. Furthermore, our approach can easily accommodate updated whole-cell net- works in accordance with rapid advancements in the protein-protein interactome and domain-domain interactions. Yet, current interactomes are still incomplete, but as suggested by Menche et al. (2015), despite this incompleteness, highly valuable disease modules were able to be constructed. Interactomes are also subject to biases from experimental methods and the degree to which a gene has been studied, but aggregative databases with high standards for inclusion help mitigate these biases (Razick et al. 2008). Personalized protein-protein interactomes that document changes owing to SNPs or somatic mutations could additionally increase the precision of individualized disease modules (Bhattacharyya et al. 2020). Other groups have mapped individual genetic profiles to protein-protein interactomes when understanding complex disease (Loscalzo 2019), but a genetics-only approach neglects to examine the downstream consequences of mutations and compensatory changes in normal genes. By including patient transcriptomics, we were able to examine how nonmutated genes are pulled into the disease module and which of these genes can be a potential therapeutic target. Individualized interactomes have been proposed, but a high-throughput method for individualized interactome assessment has yet to emerge (F Dehne and J Green, unpubl.). Such personalized advancements will further increase the accuracy and depth with which we are able to characterize individualized disease modules.

Through building this precision system biology pipeline, we uncovered key insights about patient-to-patient variability in cancer disease genes. First, we found strong evidence for the minidriver model, which postulates that there are subtle context-specific driver genes that enable individualized disease (Castro-Giner et al. 2015). Rare mutations have long been suspected to be implicated in tumor growth, but showing this at the single-patient level has previously been difficult. Clinically, extended genetic screens for tumors with atypical genetic alterations have addressed the obstacle of rare mutations (Zhu et al. 2019), but these assays neglect the context of a patient's baseline cellular environment and cooccurring mutations. To appreciate this broader context, we incorporated normal tissue transcriptomes and interacting mutation paths while building the individualized disease module. We quantify the sum cooperativity and importance of a disease gene by measuring several disease module parameters. Network parameters have been used successfully in cohort-based algorithms to prioritize drug targets and gene pathogenicity (Dunn et al. 2005; 
A

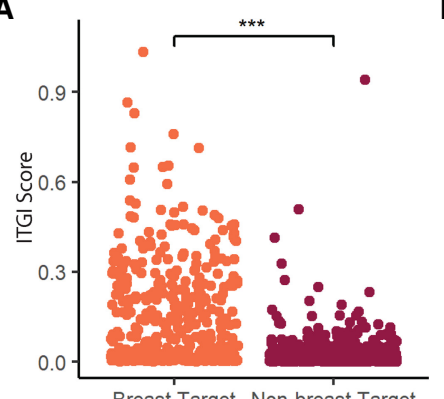

Breast Target Non-breast Target

C

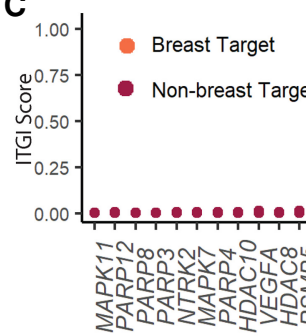

D

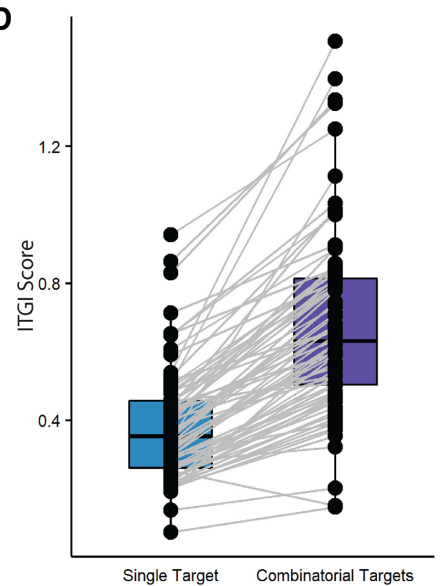

B

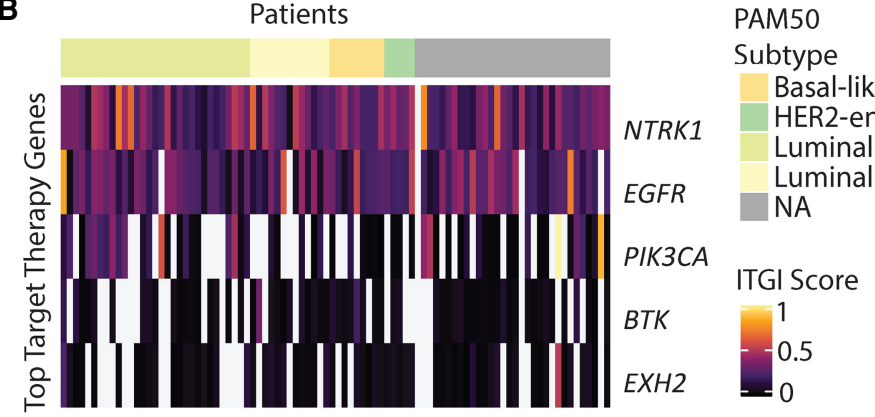

Figure 6. Individualized disease modules for formulating singular and combinatorial individualized therapies. ( $A$ ) Dot plot showing the target scoring for breast cancer versus nonbreast targets. (B) Heatmap of the highest-scoring gene targets. (C) Dot plots of targeting score for each target. (D) Paired boxplots showing the maximum single-target and maximum combinatorial target score. ( $E$ ) Drug synergy differential (synergistic - additive) for this patient's therapeutic combinations. Red bars indicate positively synergistic combination; blue bars, negatively synergistic combination. $(F)$ A single patient's top 10 combinatorial therapies scored simultaneously (synergistic) and additively removal.

Zhong et al. 2009; Boezio et al. 2017). As ground truth for minidrivers has yet to be established, the highest-accuracy disease module disruption parameters remain unknown. Despite this, we were able to perform two-step in silico validation confirming that IDGI scores recover relevant putative mini-drivers for individual patients. This is a crucial first step toward understanding pathogenic mechanisms in a patient- and tumor-specific manner.

Similarly, we revealed the interacting unmutated genes that contributed to individualized disease. Disease module studies have found that increasing numbers of diseased genes bring in more seemingly normal actors into the disease module, but until now, this has not been confirmed to apply to individual patients (Menche et al. 2015). A recent meta-analysis of patients treated with targeted or chemotherapies found that many cancers with a high mutational burden had a decreased overall survival, and this effect could only be mitigated by immunotherapies (Valero et al. 2021). In light of our findings, the negative association be- tween mutational burden and survival may reflect an increase in module size, which allows for disease resilience against traditional pharmacotherapies in combination with other factors.

Through this work, we shed light on unique disease determinates and characterize the dynamic nature of more familiar pathogenic factors. Omic studies have found distinct driver genes for BC subtypes and metastatic tumors, but deeper precision medicine contexts have been unexplored (Rajendran and Deng 2017; Bailey et al. 2018; Annunziato et al. 2019). With the unparalleled resolution shown here, we characterize the range of influence a gene can exert on an individual disease module. We describe this contextspecific variability as synthetic penetrance. Gene penetrance has traditionally been described as the percentage of individuals with a given gene variant that display a trait (Zlotogora 2003). Here, in synthetic penetrance, the "trait" is how influential a gene is in the disease module. We found that synthetic penetrance varied widely across patients, which is not surprising given the

\section{Genome Research}

www.genome.org 
diversity of mutations and disease presentations that make up the analyzed cohort. For our two most prevalent disease genes, PIK3CA and TP53, we observed distinct peaks in IDGI scores at specific levels of mutational burden. Precision system analysis with our approach can further clarify how disease module dynamics act in individuals and how synthetic penetrance can evolve over time within a tumor.

Given the persistent treatment resistance and variable response rates of many cancers, we leveraged our individualized disease modules and gene importance pipelines to extend our analysis into precision pharmacology. Targeted therapies have provided durable response rates and survival benefits in many tumors, but for others, indications for use are unclear (Murdoch and Sager 2008; De Palma et al. 2017; Xie et al. 2020). Fully using targeted therapies and other drugs for repurposing efforts requires a mechanistically informed predictive approach. We begin this effort in precision medicine by measuring the effect of targeted therapy on an individualized disease module. Additionally, we performed a combinatorial screening to identify synergistic target-therapy genes in individual patients. These results show that disease module disruption evaluation is a viable strategy for combinatorial therapy prioritization. Tuning of disease module parameter scoring from large-scale in vitro drug screening on patientderived xenographs will be required to translate this pipeline to clinical settings. To date, such data have been limited, but the in silico work presented here shows that system-informed precision oncology has immense promise.

In summary, we show a novel theoretical basis and corresponding computational pipeline for understanding individualized disease modules. Through this, we were able to show that there is an underlying phenomenon termed synthetic penetrance, which is only appreciable when we examine a disease gene across several individually characterized BC patients. Furthermore, these individualized disease modules can be functionalized to prioritize precision medicine targets. We anticipate that the principles and pipeline presented here will be able to prioritize disease genes and therapeutic targets in other complex diseases such as metabolic syndrome or neurodegenerative conditions in future work. We believe that even within monogenic diseases, this framework can reveal new insights by understanding the pathogenic variant's interactions with background patient SNPs. Because of this, we anticipate that individualized disease modules have a strong future in the field of precision medicine and pharmacology.

\section{Methods}

\section{Data acquisition and processing}

Data processing and method creation were performed in $\mathrm{R}$ (R Core Team 2021). Matched genomic and transcriptomic data corresponding to $90 \mathrm{BC}$ patients were downloaded from the TCGABRCA project (The Cancer Genome Atlas Network 2012; https ://portal.gdc.cancer.gov/projects/TCGA-BRCA). The selected patient profiles had RNA-seq data for both tumor and normal tissues and mutations annotations for tumor tissues. Patient IDs are in Supplemental Table S2. Tumor mutation annotations were available in four MAF files, with each containing mutational identification analysis from Sniper, MUTECT, VarScan, and Muse, and these data were processed using the maftools package in $\mathrm{R}$ (Koboldt et al. 2012; Larson et al. 2012; Cibulskis et al. 2013; Fan et al. 2016; Mayakonda et al. 2018). Mutations annotations from these four MAF files were aggregated for each patient and used in downstream analyses. RNA-seq data for tumor and normal BC samples were fil- tered for low-variance genes and normalized. Differential gene expression analysis was performed using the package DESeq2 (Love et al. 2014).

\section{Target-therapy data acquisition}

Target-therapy drugs were pulled from the NIH NCI TargetTherapy Fact Sheet. The gene targets of these drugs were obtained from DrugBank (Wishart et al. 2008). Drugs whose targets were not available in DrugBank, immunotherapies, and broad targeting agents were excluded. Details on these targets are in Supplemental Table S1.

\section{The PERMUTOR algorithm}

We combined the described individualized disease module construction, gene importance scoring, and target-therapy gene scoring into a complete pipeline called PERsonalized MUtation evaluaTOR (PERMUTOR). The PERMUTOR algorithm aims to prioritize the most important mutations and therapeutic targets within an individual patient's tumor according to their disruptive effects on the patient's individual disease module. Initially, an individualized disease module is created by extracting the most important shortest paths between pairs of mutations from the PPI. This is performed to distill the entire interactome down to the cooperative interactions that contain disease activity. The impact of each disease gene on the individual disease module is then evaluated by measuring the change the removal of that gene and its cooperative paths cause in key network parameters. Target therapies were also examined in this manner to find existing drugs that could be efficacious in a specific patient. The individual disease module themselves also offer biological insights.

\section{PPI preprocessing: including only expressed proteins in the generalized network}

The PPI network used in this work was generated from the irefindex database (Razick et al. 2008; http://irefindex.org/wiki/index .php?title=iRefIndex). Connections (edges/nodes) were filtered as described by Da Rocha et al. (2016). The resultant network contained 249,852 connections (edges) and 16,375 proteins (nodes). Before annotating the network with individualized -omics, genes that did not have significant expression in the patient's normal tissue sample or tumor sample were removed from the generalized network. The individual patient data from mutational profiles and RNA-seq differential expression were annotated on each patient's PPI for use in future scoring. Information from the CGC was also annotated on each patient's PPI network (Sondka et al. 2018).

\section{Stage 1: computing the shortest PPI paths that link two mutated genes in the PPI network}

Because mutated genes exert their functional effects on cancer cells via interactions with their partners in the PPI network, we hypothesized that shortest paths between two mutated genes contain disease activity. The all_shortest_paths function in the igraph package was used to compute shortest paths for each pair of mutated genes found in a single patient.

\section{Stage 2: assessment of shortest PPI paths that link two mutated genes in the PPI network}

Not all mutations or cooperative paths were important in the disease etiology of a single patient, and thus, a path-scoring scheme was created to distill the most important interactions. The shortest paths connecting pairs of mutated genes were deemed potentially 
important in individualized disease etiology if one or more of the following criteria are met: (1) the shortest paths are enriched with differentially expressed genes; (2) the paths are enriched with disease-context genes; and (3) the paths contain one or more mutated genes from that patient. As such, the Path Score was devised to assess and identify shortest paths that are potentially important.

Each gene within the path was scored, and the path score was calculated to be the sum of the components averaged across the length of the path. This formula takes into account: (1) fold change RNA-seq between tumor and tissue normal of that patient (r), (2) if the path contained nonterminal nodes that were mutated in that patient (m), and (3) a constant associated with the gene tier in the CGC (https://cancer.sanger.ac.uk/census). The CGC is a database of genes found to be highly implicated in tumorigenesis. Tier 1 genes have very strong literature support for being impactful to cancer biology, and tier 2 genes have less evidence but are still suspected to be important. A constant was added for tier 1, and a smaller constant was added for tier 2 . No constant was added if the gene was not in the CGC gene census.

$$
\text { Path Score: } p=\frac{\sum_{i=1}^{n}\left|r_{i}\right|+t_{i}+m_{i}}{n},
$$

where $\mathrm{r}=$ Fold change RNA-seq; $\mathrm{t}=\mathrm{CGC}$ tier score (tier $1=10$, tier 2 $=2) ; \mathrm{m}=$ mutation score $=5$; and $\mathrm{n}=$ path length.

To benchmark our method, paths scores were compared with the scores of 1000 random paths of the same length to calculate an empirical $P$-value for a given real path. Randomized paths were scored using the same patient's fold change and mutational data so each patient had unique sets of randomized paths scored with their omic data. The empirical $P$-value is calculated as the percentage of random paths that score higher than the real path. In this way, a significant path will have a lower empirical $P$-value and be higher than most randomized paths. All paths that have a $P$-value over a certain threshold ( $P$-value $<0.01$ in this work) were moved into a unique individualized disease module.

\section{Stage 3: construction of personalized disease modules and evaluation of the importance of disease genes via IDGI scoring}

To understand the impact of each mutated gene in the individual disease module, module parameters were measured before and after removal of a gene and its cooperative relationships. All cooperative paths that began or ended with the gene under investigation were removed from the disease module. The separated disease module components, edge number, bottlenecks, hubs, and highly trafficked hubs were measured for the altered individual disease module. The difference in each parameter between the altered and the original individual disease module was recorded for each gene. These changes in parameter values were used to calculate an IDGI score that quantified the gene's importance to the individualized disease module.

\section{Parameter definitions}

Parameter definitions are as follows:

Highly trafficked hubs: Highly trafficked hubs were defined as nodes that had edge connectivity and betweenness as measured by the betweenness function in igraph in the top fifth percentile of that disease module.

Hubs: Hubs were defined as nodes that met the edge connectivity cutoff of the fifth percentile but not the betweenness connectivity.

Bottlenecks: Bottlenecks were defined as nodes that had edge connectivity in the bottom fifth percentile of that disease module.
Separated network components: Separated network components were defined as completely disconnected subgroups within the entire disease module architecture. The count_components function from igraph was used to find the number of disconnected submodules:

$$
\begin{aligned}
\text { IDGIScore }= & 10(\Delta \text { subnetworks })+3(\Delta \text { bottlenecks })+5(\Delta \text { hubs }) \\
& +6(\Delta \text { highly trafficked hubs }) .
\end{aligned}
$$

These topological parameters were weighted $(10,3,5,6)$ to reflect their importance in network science connectivity and functionality based on previous work in the field (Al-aamri et al. 2019; Zhao and Liu 2019). These weights are also indicative of their frequency in biological networks. For example, disconnected subnetworks are rare occurrences that completely sever linkages between genes that once interacted, and because of this, disconnected subnetworks are weighted the largest because they are rare and have the most drastic effect on the network topology.

\section{Stage 4: individualized drug target analysis}

In the next phase, genes targeted by cancer target therapies were investigated. Approved targeted therapies were found via the NIH NCI Targeted Cancer Therapies website and DrugBank (Supplemental Table S1). All genes within the individualized disease module were compared to this list of targets. All targets that were represented in the disease module were removed one at a time, and the disease module was rescored after each exclusion. Next, pairs of drug targets were removed, and the disease module was rescored to find potent combinations that displayed network disruption above single-target disruption:

$$
\begin{aligned}
\text { ITGI Score }= & 10(\Delta \text { subnetworks })+3(\Delta \text { bottlenecks })+5(\Delta \text { hubs }) \\
& +6(\Delta \text { highly trafficked hubs }) .
\end{aligned}
$$

\section{Assessment of the performance of the PERMUTOR algorithm}

To validate and explore our pipeline, we constructed five testing schemes:

Test scheme 1: P-value pruning characterization. For five test patients of varying mutational burden, we constructed individualized disease modules using empirical $P$-value cutoffs of $0.005,0.01$, and 0.05 . The resulting disease modules were analyzed for their number of nodes, edges, and percentage of significant paths.

Test scheme 2: Generation of cohort disease module. Here, a cohort disease module was constructed using the well-known algorithm GRNBoost2 (Moerman et al. 2019) from the Arboreto Python package. All patient transcriptomic profiles were used as input for GRNBoost2. This disease module was pruned to include edges weighted with values greater than 25 . This cohort module was then compared to all individualized disease modules.

Test scheme 3: Individualized disease module randomization. For 10 representative patients, their individualized disease module was tested by randomly shuffling node labels 10 times and rescoring the mutated genes. These randomly shuffled scores were compared to the original score for each gene in that patient.

Test scheme 4: CGC receiver operator curve (ROC) analysis and ablation studies. ROC analysis was completed by testing the recovery of CGC genes using all patients' IDGI scores. This was repeated for CGC genes that were indicated as impactful to BC specifically. For path and IDGI score ablation studies, the five test patients were used. For each run, components of the scores were removed, and the ROC analysis was repeated.

\section{Genome Research}

www.genome.org 
Test scheme 5: Target-therapy-approved indication comparisons. Clinical information for each patient was extracted from the TCGA-BRCA project. Approved indications for each target therapy were found from DrugBank and the NIH NCI TargetTherapy Fact Sheet. First, BC-approved targets were compared against non-BC targets to show our ITGI score's ability to recapitulate cancer-specific approvals. Second, the BC-approved targets were investigated for specific subtype approvals, and patients who fit the approval criteria were compared against those who did not meet the approval indications.

\section{Figures, analysis, and website}

Schematic figures were created in BioRender (https://biorender .com/). All other figures were created in $\mathrm{R}$ and combined in Adobe Illustrator. The PERMUTOR Data Portal was created using R Shiny.

\section{Software availability}

The software generated in this study are available at GitHub (https://github.com/HuLiLab) and as Supplemental Code S1. The data generated in this study are available in the Supplemental Material and at the PERMUTOR Data Portal (https://syspharm .shinyapps.io/PERMUTOR/).

\section{Competing interest statement}

The authors declare no competing interests.

\section{Acknowledgments}

We thank James J. Collins for his suggestions on this work. This work was supported by grants from National Institutes of Health (NIH; R01CA208517, R01AG056318, R01AG61796, P50CA13 6393, R01CA240323, 5T32GM065841-1); the Glenn Foundation for Medical Research, Mayo Clinic Center for Biomedical Discovery, Center for Individualized Medicine, Mayo Clinic Cancer Center (NIH; P30CA015083), and the David F. and Margaret T. Grohne Cancer Immunology and Immunotherapy Program.

Author contributions: T.M.W., C.Y.U., C.C., C.Z., and H.L. contributed to the conception and design of the study. T.M.W., C.Y.U., C.C., C.Z., and H.L. contributed to the acquisition of data. T.M.W., C.Y.U., C.C., C.Z., and H.L. contributed to the analysis and interpretation of data. T.M.W., C.Y.U., C.C., and H.L. drafted the manuscript. H.L. supervised the study.

\section{References}

Al-aamri A, Taha K, Al-hammadi Y, Maalouf M, Homouz D. 2019. Analyzing a co-occurrence gene-interaction network to identify disease-gene association. BMC Bioinformatics 20: 70. doi:10.1186/s12859-019-2634-7

Annunziato S, de Ruiter JR, Henneman L, Brambillasca CS, Lutz C, Vaillant F, Ferrante F, Drenth AP, van der Burg E, Siteur B, et al. 2019. Comparative oncogenomics identifies combinations of driver genes and drug targets in BRCA1-mutated breast cancer. Nat Commun 10: 397. doi:10.1038/s41467-019-08301-2

Aronson SJ, Rehm HL. 2015. Building the foundation for genomics in precision medicine. Nature 526: 336-342. doi:10.1038/nature15816

Azizian NG, Li Y. 2020. XPO1-dependent nuclear export as a target for cancer therapy. J Hematol Oncol 13: 61. doi:10.1186/s13045-020-00903-4

Bailey MH, Tokheim C, Porta-Pardo E, Sengupta S, Bertrand D, Weerasinghe A, Colaprico A, Wendl MC, Kim J, Reardon B, et al. 2018. Comprehensive characterization of cancer driver genes and mutations. Cell 174: 1034-1035. doi:10.1016/j.cell.2018.07.034

Bashashati A, Haffari G, Ding J, Ha G, Lui K, Rosner J, Huntsman DG, Caldas C, Aparicio SA, Shah SP. 2012. DriverNet: uncovering the impact of somatic driver mutations on transcriptional networks in cancer Genome Biol 13: R124. doi:10.1186/gb-2012-13-12-r124

Baugh EH, Ke H, Levine AJ, Bonneau RA, Chan CS. 2018. Why are there hotspot mutations in the TP53 gene in human cancers? Cell Death Differ 25: 154-160. doi:10.1038/cdd.2017.180

Bhattacharyya R, Ha MJ, Liu Q, Akbani R, Liang H. 2020. Personalized network modeling of the pan-cancer patient and cell line interactome. JCO Clin Cancer Inform 4: 399-411. doi:10.1200/CCI.19.00140

Boezio B, Audouze K, Ducrot P, Taboureau O. 2017. Network-based approaches in pharmacology. Mol Inform 36: 1700048. doi:10.1002/ minf.201700048

Cahan P, Li H, Morris SA, Lummertz Da Rocha E, Daley GQ, Collins JJ. 2014. CellNet: network biology applied to stem cell engineering. Cell 158: 903-915. doi:10.1016/j.cell.2014.07.020

The Cancer Genome Atlas Network 2012. Comprehensive molecular portraits of human breast tumors. Nature 490: 61-70. doi:10.1038/ nature 11412

Carrasco-Ramiro F, Peiró-Pastor R, Aguado B. 2017. Human genomics projects and precision medicine. Gene Ther 24: 551-561. doi:10.1038/gt .2017 .77

Castro-Giner F, Ratcliffe P, Tomlinson I. 2015. The mini-driver model of polygenic cancer evolution. Nat Rev Cancer 15: 680-685. doi:10.1038/ nrc3999

Cerami E, Demir E, Schultz N, Taylor BS, Sander C. 2010. Automated network analysis identifies core pathways in glioblastoma. PLoS One 5: e8918. doi:10.1371/journal.pone.0008918

Chen H, Zhang Z. 2013. Prediction of associations between OMIM diseases and microRNAs by random walk on OMIM disease similarity network. Sci World J 2013: 204658. doi:10.1155/2013/204658

Chen L, Xing Z, Huang T, Shu Y, Huang G, Li H. 2016. Application of the shortest path algorithm for the discovery of breast cancer-related genes. Curr Bioinform 11: 51-58. doi:10.2174/1574893611666151119220024

Chiron M, Bagley RG, Pollard J, Mankoo PK, Henry C, Vincent L, Geslin C, Baltes N, Bergstrom DA. 2014. Differential antitumor activity of aflibercept and bevacizumab in patient-derived xenograft models of colorectal cancer. Mol Cancer Ther 13: 1636-1644. doi:10.1158/1535-7163.MCT13-0753

Cho A, Shim JE, Kim E, Supek F, Lehner B, Lee I. 2016. MUFFINN: cancer gene discovery via network analysis of somatic mutation data. Genome Biol 17: 129. doi:10.1186/s13059-016-0989-x

Cibulskis K, Lawrence MS, Carter SL, Sivachenko A, Jaffe D, Sougnez C, Gabriel S, Meyerson M, Lander ES, Getz G. 2013. Sensitive detection of somatic point mutations in impure and heterogeneous cancer samples. Nat Biotechnol 31: 213-219. doi:10.1038/nbt.2514

Codling EA, Plank MJ, Benhamou S. 2008. Random walk models in biology. $J R$ Soc Interface 5: 813-834. doi:10.1098/rsif.2008.0014

Dagogo-Jack I, Shaw AT. 2018. Tumour heterogeneity and resistance to cancer therapies. Nat Rev Clin Oncol 15: 81-94. doi:10.1038/nrclinonc.2017 .166

Da Rocha EL, Ung CY, McGehee CD, Correia C, Li H. 2016. NetDecoder: a network biology platform that decodes context-specific biological networks and gene activities. Nucleic Acids Res 44: e100. doi:10.1093/nar/ gkw166

De Palma M, Biziato D, Petrova TV. 2017. Microenvironmental regulation of tumour angiogenesis. Nat Rev Cancer 17: 457-474. doi:10.1038/nrc .2017 .51

DiNardo CD, Perl AE. 2019. Advances in patient care through increasingly individualized therapy. Nat Rev Clin Oncol 16: 73-74. doi:10.1038/ s41571-018-0156-2

Dowler S, Currie RA, Campbell DG, Deak M, Kular G, Downes CP, Alessi DR. 2000. Identification of pleckstrin-homology-domain-containing proteins with novel phosphoinositide-binding specificities. Biochem $J$ 351: $19-31$. doi: $10.1042 / b j 3510019$

Dunn R, Dudbridge F, Sanderson CM. 2005. The use of edge-betweenness clustering to investigate biological function in protein interaction networks. BMC Bioinformatics 6: 39. doi:10.1186/1471-2105-6-39

Fan Y, Xi L, Hughes DST, Zhang J, Zhang J, Futreal PA, Wheeler DA, Wang W. 2016. MuSE: accounting for tumor heterogeneity using a samplespecific error model improves sensitivity and specificity in mutation calling from sequencing data. Genome Biol 17: 178. doi:10.1186/ s13059-016-1029-6

Iborra-Egea O, Gálvez-Montón C, Roura S, Perea-Gil I, Prat-Vidal C, SolerBotija C, Bayes-Genis A. 2017. Mechanisms of action of sacubitril/valsartan on cardiac remodeling: a systems biology approach. NPJ Syst Biol Appl 3: 12. doi:10.1038/s41540-017-0013-4

Jia P, Zhao Z. 2014. VarWalker: personalized mutation network analysis of putative cancer genes from next-generation sequencing data. PLoS Comput Biol 10: e1003460. doi:10.1371/journal.pcbi.1003460

Katzav E, Nitzan M, Ben-Avraham D, Krapivsky PL, Kühn R, Ross N, Biham O. 2015. Analytical results for the distribution of shortest path lengths 
in random networks. Europhys Lett 111: 26006. doi:10.1209/0295-5075/ $111 / 26006$

Koboldt DC, Zhang Q, Larson DE, Shen D, McLellan MD, Lin L, Miller CA Mardis ER, Ding L, Wilson RK. 2012. VarScan 2: somatic mutation and copy number alteration discovery in cancer by exome sequencing. Genome Res 22: 568-576. doi:10.1101/gr.129684.111

Kuijjer ML, Tung MG, Yuan GC, Quackenbush J, Glass K. 2019. Estimating sample-specific regulatory networks. iScience 14: 226-240. doi:10.1016/ j.isci.2019.03.021

Larson DE, Harris CC, Chen K, Koboldt DC, Abbott TE, Dooling DJ, Ley TJ, Mardis ER, Wilson RK, Ding L. 2012. SomaticSniper: identification of somatic point mutations in whole genome sequencing data. Bioinformatics 28: 311-317. doi:10.1093/bioinformatics/btr665

Lin M, Ye M, Zhou J, Wang ZP, Zhu X. 2019. Recent advances on the molecular mechanism of cervical carcinogenesis based on systems biology technologies. Comput Struct Biotechnol J 17: 241-250. doi:10.1016/j .csbj.2019.02.001

Loscalzo J. 2019. Precision medicine: a new paradigm for diagnosis and management of hypertension? Circ Res 124: 987-989. doi:10.1161/ CIRCRESAHA.119.314403

Love MI, Huber W, Anders S. 2014. Moderated estimation of fold change and dispersion for RNA-seq data with DESeq2. Genome Biol 15: 550. doi:10.1186/s13059-014-0550-8

Luck K, Kim D, Lambourne L, Spirohn K, Begg BE, Bian W, Brignall R, Cafarelli T, Campos-laborie FJ, Charloteaux B, et al. 2020. A reference map of the human binary protein interactome. Nature 580: 402-408. doi:10.1038/s41586-020-2188-x

Managbanag JR, Witten TM, Bonchev D, Fox LA, Tsuchiya M, Kennedy BK, Kaeberlein M, Lehner B. 2008. Shortest-path network analysis is a useful approach toward identifying genetic determinants of longevity. PLoS One 3: e3802. doi:10.1371/journal.pone.0003802

Mayakonda A, Lin DC, Assenov Y, Plass C, Koeffler HP. 2018. Maftools: efficient and comprehensive analysis of somatic variants in cancer. Genome Res 28: 1747-1756. doi:10.1101/gr.239244.118

Menche J, Sharma A, Kitsak M, Ghiassian SD, Vidal M, Loscalzo J, Barabasi AL. 2015. Uncovering disease-disease relationships through the incomplete interactome. Science 347: 1257601. doi:10.1126/science.1257601

Moerman T, Santos SA, Gonza CB, Simm J, Moreau Y, Aerts J, Aerts S. 2019. Gene expression GRNBoost2 and Arboreto: efficient and scalable inference of gene regulatory networks. Bioinformatics 35: 2159-2161. doi:10 $.1093 /$ bioinformatics/bty916

Murdoch D, Sager J. 2008. Will targeted therapy hold its promise? An evidence-based review. Curr Opin Oncol 20: 104-111. doi:10.1097/CCO .0b013e3282f44b12

Oh JH, Jang SJ, Kim J, Sohn I, Lee JY, Cho EJ, Chun SM, Sung CO. 2020. Spontaneous mutations in the single TTN gene represent high tumor mutation burden. NPJ Genomic Med 5: 33. doi:10.1038/s41525-019$0107-6$

O'Neil NJ, Bailey ML, Hieter P. 2017. Synthetic lethality and cancer. Nat Rev Genet 18: 613-623. doi:10.1038/nrg.2017.47

Rajendran BK, Deng CX. 2017. Characterization of potential driver mutations involved in human breast cancer by computational approaches. Oncotarget 8: 50252-50272. doi:10.18632/oncotarget.17225

Razick S, Magklaras G, Donaldson IM. 2008. iRefIndex: a consolidated protein interaction database with provenance. BMC Bioinformatics 9: 405 doi:10.1186/1471-2105-9-405

R Core Team. 2021. R: a language and environment for statistical computing. $\mathrm{R}$ Foundation for Statistical Computing, Vienna. https://www.r-project .org/.

Relling MV, Evans WE. 2015. Pharmacogenomics in the clinic. Nature 526: 343-350. doi:10.1038/nature15817

Reyna MA, Leiserson MDM, Raphael BJ. 2018. Hierarchical HotNet: identifying hierarchies of altered subnetworks. Bioinformatics 34: i972-i980 doi:10.1093/bioinformatics/bty613
Sharma A, Halu A, Decano JL, Padi M, Liu YY, Prasad RB, Fadista J, Santolini $\mathrm{M}$, Menche J, Weiss ST, et al. 2018. Controllability in an islet specific regulatory network identifies the transcriptional factor NFATC4, which regulates type 2 diabetes associated genes. NPJ Syst Biol Appl 4: 25. doi:10.1038/s41540-018-0057-0

Sondka Z, Bamford S, Cole CG, Ward SA, Dunham I, Forbes SA. 2018. The COSMIC Cancer Gene Census: describing genetic dysfunction across all human cancers. Nat Rev Cancer 18: 696-705. doi:10.1038/s41568018-0060-1

Valero C, Lee M, Hoen D, Wang J, Nadeem Z, Patel N, Postow MA, Shoushtari AN, Plitas G, Balachandran VP, et al. 2021. The association between tumor mutational burden and prognosis is dependent on treatment context. Nat Genet 53: 11-15. doi:10.1038/s41588-020-00752-4

Werner RJ, Kelly AD, Issa JPJ. 2017. Epigenetics and precision oncology. Cancer J 23: 262-269. doi:10.1097/PPO.0000000000000281

Wishart DS, Knox C, Guo AC, Cheng D, Shrivastava S, Tzur D, Gautam B, Hassanali M. 2008. DrugBank: a knowledgebase for drugs, drug actions and drug targets. Nucleic Acids Res 36(suppl_1): D901-D906. doi:10 $.1093 / \mathrm{nar} / \mathrm{gkm} 958$

Wuchty S. 2001. Scale-free behavior in protein domain networks. Mol Biol Evol 18: 1694-1702. doi:10.1093/oxfordjournals.molbev.a003957

Xia F, Liu J, Nie H, Fu Y, Wan L, Kong X. 2020. Random walks: a review of algorithms and applications. IEEE Trans Emerg Top Comput Intell 4 95-107. doi:10.1109/TETCI.2019.2952908

Xie YH, Chen YX, Fang JY. 2020. Comprehensive review of targeted therapy for colorectal cancer. Signal Transduct Target Ther 5: 22. doi:10.1038/ s41392-020-0116-z

Xu C, Li X, Liu P, Li M, Luo F. 2019. Patient-derived xenograft mouse models: a high fidelity tool for individualized medicine (review). Oncol Lett 17: 3-10. doi:10.3892/ol.2018.9583

Yadav A, Vidal M, Luck K. 2020. Precision medicine: networks to the rescue. Curr Opin Biotechnol 63: 177-189. doi:10.1016/j.copbio.2020.02.005

Yu H, Kim PM, Sprecher E, Trifonov V, Gerstein M. 2007. The importance of bottlenecks in protein networks: correlation with gene essentiality and expression dynamics. PLoS Comput Biol 3: e59. doi:10.1371/journal.pcbi .0030059

Zagidullin B, Aldahdooh J, Zheng S, Wang W, Wang Y, Saad J, Malyutina A, Jafari M, Tanoli Z, Pessia A, et al. 2019. DrugComb: an integrative cance drug combination data portal. Nucleic Acids Res 47: W43-W51. doi:10 $.1093 /$ nar/gkz337

Zhao S, Iyengar R. 2012. Systems pharmacology: network analysis to identify multiscale mechanisms of drug action. Annu Rev Pharmacol Toxicol 52: 505-512. doi:10.1146/annurev-pharmtox-010611-134520

Zhao X, Liu Z. 2019. Analysis of topological parameters of complex disease genes reveals the importance of location in a biomolecular network. Genes (Basel) 10: 143. doi:10.3390/genes10020143

Zhong Q, Simonis N, Li QR, Charloteaux B, Heuze F, Klitgord N, Tam S, Yu H, Venkatesan K, Mou D, et al. 2009. Edgetic perturbation models of human inherited disorders. Mol Syst Biol 5: 321. doi:10.1038/msb.2009.80

Zhu J, Tucker M, Marin D, Gupta RT, Healy P, Humeniuk M, Jarvis C, Zhang T, McNamara M, George DJ, et al. 2019. Clinical utility of FoundationOne tissue molecular profiling in men with metastatic prostate cancer. Urol Oncol Semin Orig Investig 37: 813.e1-813.e9. doi:10 $.1016 /$ j.urolonc.2019.06.015

Zielinski DC, Jamshidi N, Corbett AJ, Bordbar A, Thomas A, Palsson BO. 2017. Systems biology analysis of drivers underlying hallmarks of cancer cell metabolism. Sci Rep 7: 41241. doi:10.1038/srep41241

Zlotogora J. 2003. Penetrance and expressivity in the molecular age. Genet Med 5: 347-352. doi:10.1097/01.GIM.0000086478.87623.69

Received June 14, 2021; accepted in revised form November 30, 2021.

\section{Genome Research}

www.genome.org 


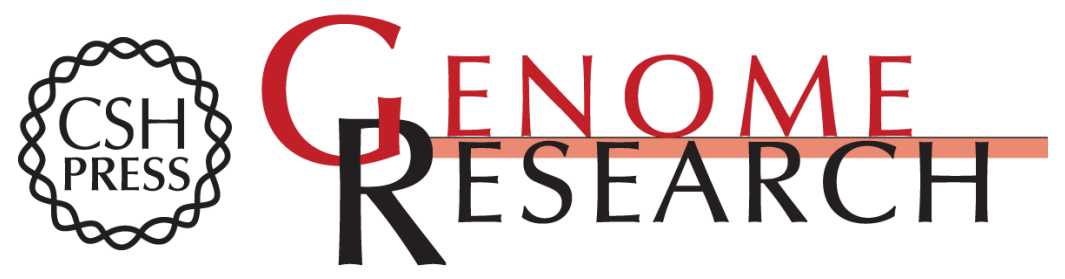

\section{De novo individualized disease modules reveal the synthetic penetrance of genes and inform personalized treatment regimens}

Taylor M. Weiskittel, Choong Y. Ung, Cristina Correia, et al.

Genome Res. 2022 32: 124-134 originally published online December 7, 2021

Access the most recent version at doi:10.1101/gr.275889.121

Supplemental Material

References

Open Access

Creative Commons

License

Email Alerting Service
http://genome.cshlp.org/content/suppl/2021/12/27/gr.275889.121.DC1

This article cites 66 articles, 6 of which can be accessed free at: http://genome.cshlp.org/content/32/1/124.full.html\#ref-list-1

Freely available online through the Genome Research Open Access option.

This article, published in Genome Research, is available under a Creative Commons License (Attribution-NonCommercial 4.0 International), as described at http://creativecommons.org/licenses/by-nc/4.0/.

Receive free email alerts when new articles cite this article - sign up in the box at the top right corner of the article or click here.

\section{Affordable, Accurate Sequencing.}

